\title{
Transcutaneous Carbon Dioxide Treatment Affects Heart Rate Variability - A Pilot Study
}

\author{
ZITA KRESKA ${ }^{1}$, BALÁZS NÉMETH ${ }^{1,2}$, ISTVÁN KISS ${ }^{2}$, IVÁN PÉTER ${ }^{1}$, ZÉNÓ AJTAY ${ }^{1,3}$ and LÁSZLÓ HEJJEL ${ }^{3}$ \\ ${ }^{I}$ Zsigmondy Vilmos SPA Hospital, Harkány, Hungary; \\ ${ }^{2}$ Department of Public Health Medicine, and ${ }^{3}$ Heart Institute, Medical School, University of Pécs, Pécs, Hungary
}

\begin{abstract}
Background: The aim of this study was to investigate the effects of carbon-dioxide treatment on heart rate variability (HRV) parameters: mean RR interval (RRI), standard deviation of $R R$ intervals $(S D N N)$, root mean square of successive $R R$ differences (RMSSD); and Porta and Guzik indices, as measures of heart rate asymmetry. Materials and Methods: Twenty patients were enrolled (mean $\pm S D$, age $=59 \pm 7.8$ years). Measurements were performed before $\mathrm{CO}_{2}$ treatment, at the beginning of treatment, at 15 min of treatment, immediately after and $1 \mathrm{~h}$ after the treatment. Results: Significant increase in SDNN was found $1 \mathrm{~h}$ after the treatment when compared to that before it ( $p=0.011)$. There were no significant changes in other parameters. Conclusion: $\mathrm{CO}_{2}$ treatment can influence the autonomic nervous system identified by SDNN changes. However, larger studies are required to confirm these results.
\end{abstract}

The transcutaneous administration of carbon dioxide $\left(\mathrm{CO}_{2}\right)$, hereafter referred to as ' $\mathrm{CO}_{2}$ treatment' has been used for curative purposes for centuries. The first paper investigating the medicinal use of $\mathrm{CO}_{2}$ was published by Brandi et al. in 1932 (1). $\mathrm{CO}_{2}$ passes freely through membranes and has a well-known vasodilatory effect. Both in vitro and in vivo studies have demonstrated a rightward shift of the oxygenhaemoglobin dissociation curve after administration of $\mathrm{CO}_{2}$. Sakai et al. described this as an "artificial Bohr effect". This is responsible for the decrease in $\mathrm{pH}$ and increase in partial pressure of oxygen in the tissues, through facilitated $\mathrm{O}_{2}$ release, which were shown in vivo (2). The findings of Minamiyama and Yamamoto confirmed these effects using intra-vital video microscopy to demonstrate subcutaneous

This article is freely accessible online.

Correspondence to: Dr. László Hejjel, Heart Institute, Medical School, University of Pécs, Ifjúság rd. 13. H-7624 Pécs, Hungary. Tel: +36 72536000, e-mail: hejjel.laszlo@pte.hu

Key Words: Carbon dioxide treatment, rehabilitation, balneotherapy, heart rate variability. vasodilation after $\mathrm{CO}_{2}$ administration. In addition, $\mathrm{CO}_{2}$ was shown to increase blood flow in the observed subcutaneous vessels (3). $\mathrm{CO}_{2}$ treatment is used to cure several diseases, such as peripheral arterial and venous disorders (e.g. claudication, and lower limb ulcer), heart diseases (e.g. hypertension, and heart failure) and immunological disorders (e.g. Raynaud's syndrome) $(4,5)$. In short, $\mathrm{CO}_{2}$ treatment is a non-invasive, highly efficient, low-cost treatment capable of easing the symptoms of arterial and venous diseases possibly due to vasodilatation and reduction of oxidative stress. However, as far as we are aware of, there is no evidence for alteration of the autonomic nervous system after $\mathrm{CO}_{2}$ treatment.

Heart rate variability (HRV) is controlled by the sympathetic and parasympathetic nervous systems, and is influenced by several other factors (e.g. autocrine, paracrine, endocrine, and mechanical stretch). The sinus node is the final summing element in controlling the heart rate. HRV analysis is a non-invasive method providing information on the autonomic regulation of cardiac activity $(6,7)$. In recent decades, several studies have shown an association between cardiovascular diseases and HRV. HRV analysis is considered a useful tool for predicting the risk of sudden cardiac death after myocardial infarction $(8,9)$. Numerous studies have investigated HRV among diabetic patients: the most useful finding is that reduced HRV can indicate diabetic neuropathy before clinical symptoms actually appear (10-12). A recent study suggested that HRV can be a potential new tool to predict complications after cardiac surgery (13). In short, HRV assessment is a non-invasive method used to assess the heart's ability to respond and adapt to external or internal stress.

The aim of this study was to investigate the instantaneous effects of a single $\mathrm{CO}_{2}$ treatment on $\mathrm{HRV}$ and heart rate asymmetry (HRA) parameters.

\section{Materials and Methods}

The present study was performed at our ISO 9001-accredited Cardiology Rehabilitation Inpatient Unit from July 2017 to January 2018 in Zsigmondy Vilmos SPA Hospital, Harkány. Non- 
diabetic, abstinent, hypertensive patients, suffering from coronary heart disease were enrolled. Patients who had previously received $\mathrm{CO}_{2}$ treatment were excluded. Moreover, patients who had suffered myocardial infarction, stroke or had undergone open heart surgery less than a year before the study were also excluded. Additionally, individuals diagnosed with any kind of cancer or kidney injury were also not enrolled. Informed written consent was obtained from each patient. The study protocol was approved by the Regional Ethics Committee at the University of Pécs, Pécs, Hungary (Permission No. 5919), in accordance with the 2008 Helsinki declaration.

$\mathrm{CO}_{2}$ gas was administered for 35 minutes in a plastic bag sealed at mid-thoracic level, as previously described by Fabry et al. (4). HRV data acquisition was performed using a hand-held, batterypowered device based on a PIC18F46J50 microcontroller (Microchip Technology Inc., Chandler, AZ, USA) and INA333 instrumentation amplifier chip (Texas Instruments, Dallas, TX, USA). The device contains analogue circuitry for a single-channel (three-electrode) electrocardiograph (ECG) amplifier among others. The recordings were transferred to a PC on an SD card for analysis. After extracting the 1-ms temporal resolution ECG signal from the stored data, automatic detection of RR intervals and manual checking was performed by ECGRdet v2.1 software (L. Hejjel, Pécs, Hungary). A 300-s-long artefact-free part of the recording was then selected manually. Particular attention was paid to selecting a portion containing exclusively normal sinus interbeat intervals in order to obtain correct parameters of HRV. Varian v2.2 software (L. Hejjel, Pécs, Hungary) was used to calculate the standard timedomain parameters: mean RR interval (meanRRI), standard deviation of normal-to-normal RR intervals (SDNN) and root mean square of successive RR differences (RMSSD), as well as HRA parameters: Porta index and Guzik index. The hardware and software (ECGRdet and Varian) were developed by L. Hejjel (1416). Non-invasive blood pressure measurements were made in the middle of each of the five stages of the study. Average respiration frequency was calculated by fast Fourier transformation of the tachogram by Varian v2.2.

During the 3-week-long in-ward rehabilitation, patients underwent a combination of physiotherapies, and received nine $\mathrm{CO}_{2}$ treatment (35 min per session). In order to follow the changes of HRV parameters, analysis was performed immediately before $\mathrm{CO}_{2}$ treatment, immediately after the beginning of the $\mathrm{CO}_{2}$ treatment, $15 \mathrm{~min}$ after the beginning of the $\mathrm{CO}_{2}$ treatment, immediately after the end of the $\mathrm{CO}_{2}$ treatment, and $1 \mathrm{~h}$ after the end of the $\mathrm{CO}_{2}$ treatment. All the measurements were performed with the patients in supine position $15 \mathrm{~min}$ after orthostatic adaptation and from $8 \mathrm{am}$ to $10 \mathrm{pm}$ in order to avoid positional or diurnal influences, respectively. The patients did not receive any medication besides their regular antihypertensive drugs.

Statistical analysis. Statistical analysis was carried out with StatistiXL package (Broadway-Nedlands, Western Australia) in MS Excel (Microsoft Corp., Redmond, WA, USA). Calculated HRV and HRA measures were assessed by Friedman test and post-hoc Wilcoxon test with Holm-Bonferroni correction. The 2-5th stages were compared to the first one. Before correction $p$-values of less than 0.05 were considered statistically significant. Charts were constructed using OriginPro 2017 (OriginLab Corp., Northampton, MA, USA).

\section{Results}

Twenty-three patients were enrolled, out of whom three were excluded due to inappropriate ECG signal quality, resulting in 20 patients remaining in the study (age, mean $\pm \mathrm{SD}=59 \pm 7.8$ years). Clinical characteristics of the participants are shown in Table I.

During the entire measurement, there were no significant systolic or diastolic blood pressure changes detected in any patient.

Regarding the meanRRI, there were no significant changes found (Figure 1A). A significant increase in SDNN was detected $1 \mathrm{~h}$ after the $\mathrm{CO}_{2}$ treatment (Friedman test: $p=0.006$, Wilcoxon test: $p=0.011$ ) (Figure 1B). No significant changes were demonstrated in the RMSSD parameter (Figure 1C).

Regarding the Porta and Guzik indices, no statistically significant differences were found during the monitoring. The effects of $\mathrm{CO}_{2}$ treatment on Porta and Guzik indices are shown in Figure 2.

The detailed results for HRV parameters measured before, during and after $\mathrm{CO}_{2}$ treatment are shown in Table II.

The breathing frequency dropped from 0.25 to $0.22 \mathrm{~Hz}$ after $15 \mathrm{~min}$ of treatment and elevated to $0.27 \mathrm{~Hz}$ immediately after $\mathrm{CO}_{2}$ treatment (Figure 3A). There was no correlation (insignificant $p$-values) between respiration frequency and HRA parameters (Figure 3B).

\section{Discussion}

In the present study, the effects of $\mathrm{CO}_{2}$ treatment on the autonomic nervous system were investigated using HRV and HRA analysis. Our protocol was aimed at minimizing the effects of diurnal changes, body position, vocalization and movements on HRV and HRA parameters. To rule out interobserver errors, all measurements were performed by the same investigator.

To the best of our knowledge this is the first study investigating the effects of $\mathrm{CO}_{2}$ treatment on $\mathrm{HRV}$ and HRA parameters.

The meanRRI remained stable during the study, reflecting steady state or balanced sympathetic and parasympathetic actions. SDNN showed a mild but continuous increase during the study, which reached statistical significance $1 \mathrm{~h}$ after the $\mathrm{CO}_{2}$ treatment by post-hoc test. Since SDNN reveals global variability including both sympathetic and parasympathetic effects $(6,17)$, some autonomic influence occurs in spite of the steady-state meanRRI. The beat-to-beat variability parameter RMSSD is a measure of vagal actions on heart rate $(6,17)$. At the beginning of the treatment a slight drop was seen, followed by a monotonous increase not reaching the level of significance. This probably occurred due to the complex cardiovascular actions of $\mathrm{CO}_{2}$, the high inter-individual variation and the relatively small sample 

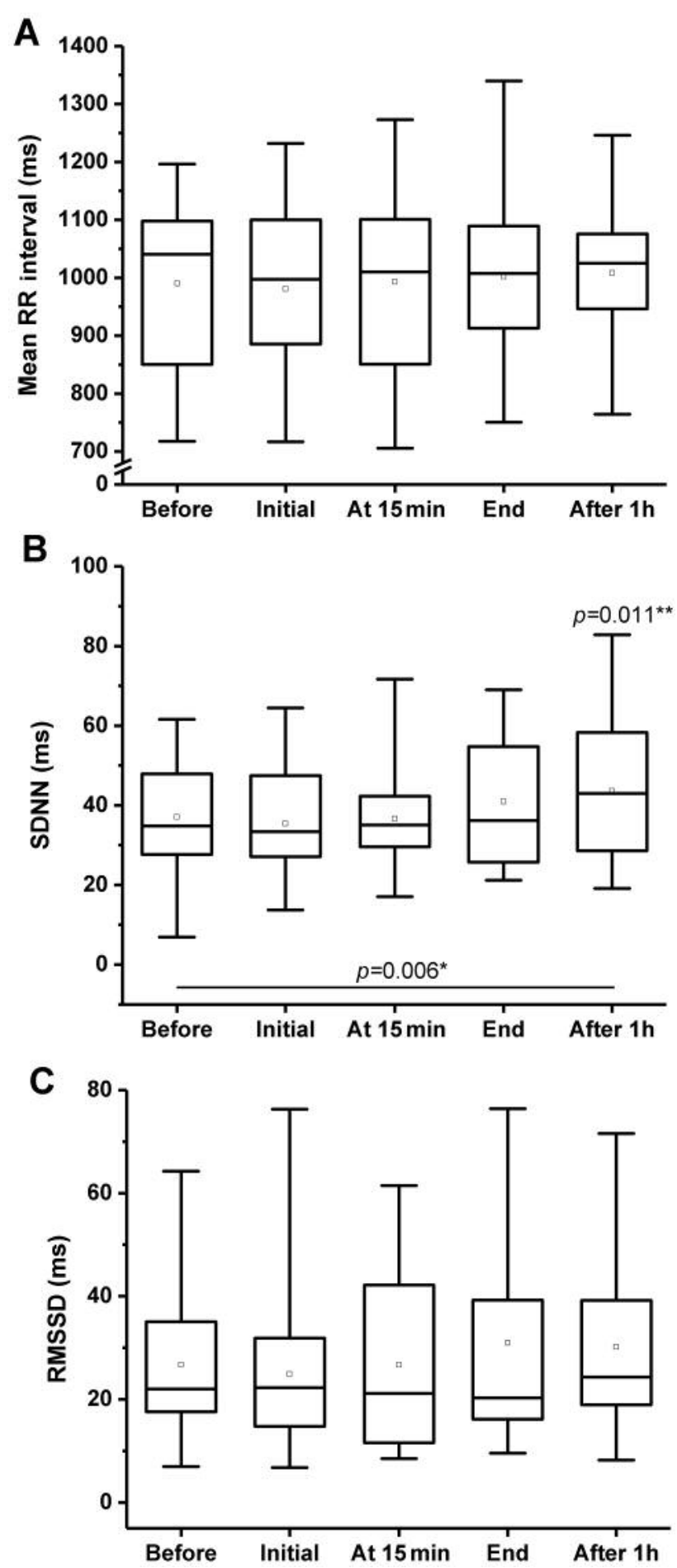

Figure 1. Time domain measurements before, during (at beginning and at $15 \mathrm{~min}$ ), and after (immediately and $1 \mathrm{~h}$ later) $\mathrm{CO}_{2}$ treatment. A: Mean time between two consecutive $R$-waves on the ECG (RR interval). B: Standard deviation of RR intervals (SDNN). C: Root mean square of successive RR interval differences (RMSSD). Boxes: Lower and upper quartiles, horizontal line: median value, whiskers: minimum and maximum values, square: mean value. *Friedman test p-value, **post-hoc Wilcoxon's paired sample test p-values, only significant differences are indicated.
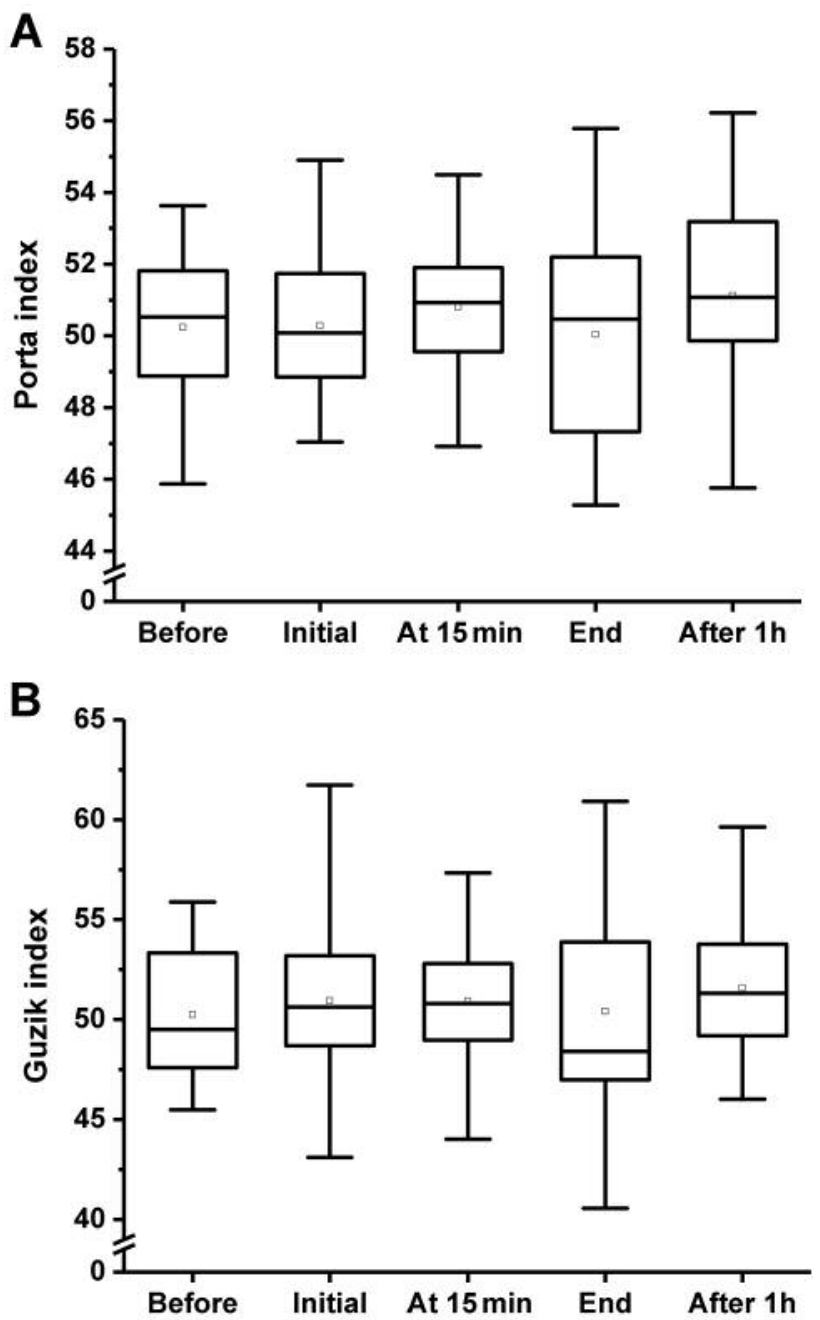

Figure 2. The effect of $\mathrm{CO}_{2}$ treatment on Porta (A) and Guzik (B) indices before, during (at the beginning and at $15 \mathrm{~min}$ ), and after (immediately and $1 \mathrm{~h}$ later) $\mathrm{CO}_{2}$ treatment. Boxes: Lower and upper quartiles, horizontal line: median value, whiskers: minimum and maximum values, square: mean value. Friedman test resulted in $p=0.555$ for both parameters.

Table I. Clinical characteristics of the participants.

\begin{tabular}{lc}
\hline & Patient group $(\mathrm{n}=20)$ \\
\hline Mean age, years $( \pm \mathrm{SD})$ & $59( \pm 7.8)$ \\
Male, $\mathrm{n}(\%)$ & $13(62)$ \\
Diabetes type 2, $\mathrm{n}(\%)$ & $4(19)$ \\
Smoker, $\mathrm{n}(\%)$ & $3(14)$ \\
$\mathrm{EF} \%(\mathrm{mean} \pm \mathrm{SD})$ & $58( \pm 4.2)$ \\
$\mathrm{BMI}, \mathrm{kg} / \mathrm{m}^{2}(\mathrm{mean} \pm \mathrm{SD})$ & $27,4( \pm 5.1)$ \\
$\mathrm{WBC} \mathrm{G} / \mathrm{I},(\operatorname{mean} \pm \mathrm{SD})$ & $7.2( \pm 1.7)$ \\
$\mathrm{CRP} \mathrm{mg} / \mathrm{l}(\operatorname{mean} \pm \mathrm{SD})$ & $5.3( \pm 1.9)$ \\
$\mathrm{HGB} \mathrm{mg} / \mathrm{l}(\operatorname{mean} \pm \mathrm{SD})$ & $136.2( \pm 11.6)$
\end{tabular}

EF: Left ventricular ejection fraction, BMI: body mass index, WBC: white blood cell count, CRP: C-reactive protein level, HGB: haemoglobin level. 

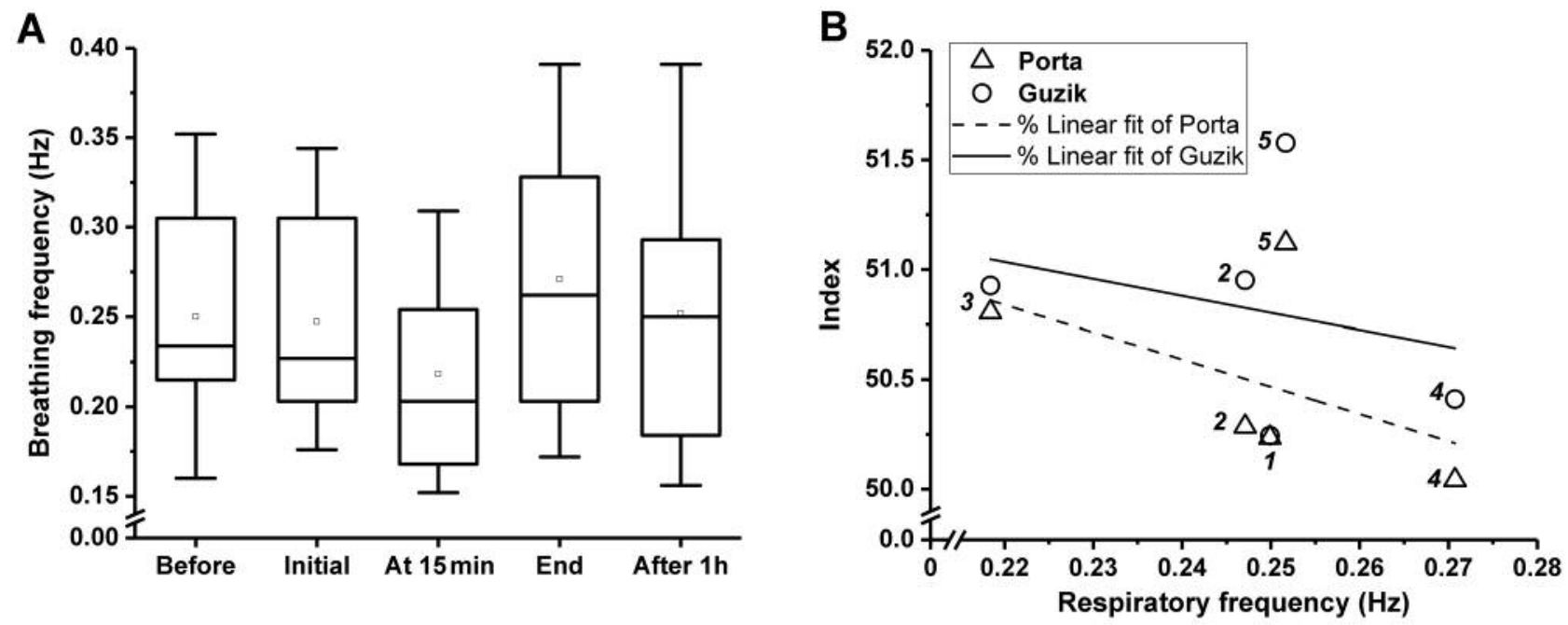

Figure 3. A: Mean values of breathing frequency derived from spectral analysis of the tachograms. Boxes: Lower and upper quartiles, horizontal line: median value, whiskers: minimum and maximum values, square: mean value. Friedman test resulted in $p=0.555$. B: Correlation of breathing frequency and heart rate asymmetry parameters with non-significant linear fit. The Arabic numbers in Italics next to data points correspond to the five measurements made. The wide divergence of the Porta and Guzik indices at the beginning of the treatment, immediately after and $1 \mathrm{~h}$ after the treatment is remarkable, as well as the elevated indices at the 5th measurement compared to the 1st and 2nd in spite of the similar breathing frequency.

Table II. Detailed results of measurements.

\begin{tabular}{lccccc}
\hline & \multicolumn{3}{c}{ Timepoint relative to $\mathrm{CO}_{2}$ treatment } \\
\cline { 2 - 6 } & Before & At start & After 15 min & After end & $1 \mathrm{~h}$ After \\
\hline Mean RRI (ms) & $989.8 \pm 130.3$ & $985.1 \pm 131.87$ & $993.7 \pm 138.8$ & $1005.8 \pm 136.8$ & $989.8 \pm 232.3$ \\
SDNN (ms) & $35.6 \pm 13.1$ & $34.1 \pm 13.8$ & $35.9 \pm 13.3$ & $40.4 \pm 16.1$ & $\mathbf{4 2 . 6 \pm 1 8 . 5}$ \\
RMSSD (ms) & $25.1 \pm 15.2$ & $23.7 \pm 16.2$ & $25.4 \pm 16.8$ & $29.7 \pm 20.6$ & $28.1 \pm 17.8$ \\
Porta index & $50.3 \pm 2.1$ & $50.2 \pm 1.6$ & $51.0 \pm 1.8$ & $49.9 \pm 3$ & $51.3 \pm 2.6$ \\
Guzik index & $50.4 \pm 3.5$ & $50.6 \pm 4.1$ & $51.7 \pm 3.5$ & $50.1 \pm 5.1$ & $52.13 \pm 3.1$ \\
\hline
\end{tabular}

MeanRRI: Mean interval between two consecutive R-waves on the ECG, SDNN: standard deviation of RRI. RMSSD: root mean square of successive RR interval differences. Bold numbers correspond to values significantly different from that before treatment after Holm-Bonferroni correction $(p=0.011)$.

size. Consequently, an increase in both sympathetic and parasympathetic activity can occur during $\mathrm{CO}_{2}$ treatment, resulting in unchanged heart rate, systolic and diastolic blood pressure. In line with our previous finding, the changes in overall HRV suggest that $\mathrm{CO}_{2}$ is not simply a vasodilator but is capable of altering the function of the cardiovascular system, possibly by activating endogenous mechanisms leading to vasodilatation (18). Bickel et al. found increased sympathetic activity during positive pressure $\mathrm{CO}_{2}$ pneumoperitoneum by HRV analysis possibly due to hypercarbia and tension of the peritoneum; however, in the present study there was no peritoneal tension (19).
Regarding the Porta and Guzik indices, no statistically significant differences were found during the investigation, once again probably due to the small sample size. However, an increase in both these indices at the end of the treatment and a mild drop immediately after the treatment was observed, although they remained higher even after $1 \mathrm{~h}$ compared to the basal level (Table II). According to our previous study, the Porta and Guzik indices are strongly correlated to each other in healthy volunteers even at different inspiration/expiration ratios (16), whereas in the current investigation, their divergence was observed at the beginning of $\mathrm{CO}_{2}$ treatment, immediately after and $1 \mathrm{~h}$ after the 
treatment (Figure 3B). The physiopathology of HRA parameters is not well known; therefore, we extracted the average breathing frequency from the tachograms by fast Fourier analysis in order to link the observed HRA changes to breathing rate. Faster breathing corresponds to a shorter expiration period relative to inspiration, theoretically resulting in higher HRA indices, based on the work of Klintworth et al. (16). On the contrary, in our study, an opposing change of breathing frequency compared with Porta and Guzik indices was observed (Figure 3B), resulting in a negative regression line. Unfortunately, the correlation was not significant due to the outlier at the final measurement, when in spite of the normalization of the breathing rate, the HRA indices further increased (Figure 3B). This phenomenon again reflects both an instantaneous and a sustained vegetative action of $\mathrm{CO}_{2}$ treatment, which cannot be explained by our monitored or computed parameters, therefore precise respiration monitoring (via transthoracic impedance or a sensitive chest strap) is recommended in subsequent investigation. This mighty be explained at the second measurement of $\mathrm{CO}_{2}$ treatment by the Bohr effect, when enough $\mathrm{CO}_{2}$ accumulates in the body to facilitate $\mathrm{O}_{2}$ release from haemoglobin $(2,3)$, resulting in slower breathing as a response to higher oxygen tension in the chemoreceptor area. After finishing the treatment, the rapid diffusion and exhalation of $\mathrm{CO}_{2}$ causes the Bohr effect to cease, which stimulates the breathing centre, resulting in a transient higher breathing immediately after the treatment (Figure 3A).

The relatively small number of participants may be the reason for the insignificant differences in RMSSD and HRA parameters.

In conclusion, the reported study protocol is adequate for investigating the effects of $\mathrm{CO}_{2}$ treatment on HRV and HRA parameters. According to the results of this pilot study, $\mathrm{CO}_{2}$ treatment can influence the autonomic nervous system. However, larger studies with respiration monitoring are required to confirm these results and to discover the mechanism.

\section{Conflict of Interest}

The Authors declare that they have no conflict of interest in regard to this study.

\section{Funding}

OriginPro v2017 software was funded by 3/2016 AOK-KA grant from the Medical Faculty of the University of Pécs, Hungary. Balázs Németh was supported by the ÚNKP-17-3-III New National Excellence Program of the Ministry of Human Capacities, Hungary.

\section{Ethical Approval}

All procedures performed in the study were in accordance with the ethical standards of the Regional Research Ethics Committee and with the 1964 Helsinki declaration and its later amendments. The study protocol was approved by the Regional Ethics Committee of University of Pécs, Pécs, Hungary (Permission No.: 5919.)

\section{Acknowledgements}

The Authors express their special thanks to all the nurses of the Cardiology Ward for their invaluable help during this study. The present scientific contribution is dedicated to the 650th anniversary of the foundation of the University of Pécs, Hungary.

\section{References}

1 Brandi C, Aniello CD, Grimaldi L, Bosi B, Dei I, Lattarulo P and Alessandrini $\mathrm{C}$ : Carbon dioxide therapy in the treatment of localized adiposities: Clinical study and histopathological correlations. Aesthetic Plast Surg 25: 170-174, 2001.

2 Sakai Y, Miwa M, Oe K, Ueha T, Koh A, Niikura T, Iwakura T, Lee SY, Tanaka M and Kurosaka M: A novel system for transcutaneous application of carbon dioxide causing an "Artificial Bohr Effect" in the human body. PLoS One 6: e24137, 2011.

3 Minamiyama $M$ and Yamamoto A: Direct evidence of the vasodilator action of carbon dioxide on subcutaneous microvasculature in rats by use of intra-vital. J Biorheol 24: 42-46, 2010.

4 Fabry R, Monnet P, Schmidt J, Lusson JR, Carpentier PH, Baguet JC and Dubray C: Clinical and microcirculatory effects of transcutaneous $\mathrm{CO}_{2}$ therapy in intermittent claudication. Randomized double-blind clinical trial with a parallel design. Vasa 38: 213-224, 2009.

5 Schmidt J, Monnet P, Normand B and Fabry R: Microcirculatory and clinical effects of serial percutaneous application of carbon dioxide in primary and secondary Raynaud's phenomenon. Vasa 34: 93-100, 2005.

6 Hejjel L and Gál I: Heart rate variability analysis. Acta Physiol Hung 88: 219-30, 2001.

7 Rajendra Acharya U, Paul Joseph K, Kannathal N, Lim CM and Suri JS: Heart rate variability: a review. Med Biol Eng Comput 44: 1031-1051, 2006.

8 Lombardi F, Mäkikallio TH, Myerburg RJ and Huikuri HV: Sudden cardiac death: Role of heart rate variability to identify patients at risk. Cardiovase Res 50: 210-217, 2001.

9 Huikuri HV, Mäkikallio TH, Raatikainen MJ, Perkiömäki J, Castellanos A and Myerburg RJ: Prediction of sudden cardiac death: Appraisal of the studies and methods assessing the risk of sudden arrhythmic death. Circulation 108: 110-115, 2003.

10 Singh JP, Larson MG, O’Donell CJ, Wilson PF, Tsuji H, LyodJones DM and Levy D: Association of hyperglycemia with reduced heart rate variability: the Framingham heart study. Am J Cardiol 86: 309-312, 2003.

11 Wheeler T and Watkins PJ: Cardiac denervation in diabetes. $\mathrm{Br}$ Med J 4: 584-586, 1973.

12 Istenes I, Körei AE, Putz Z, Németh N, Martos T, Keresztes K, Kempler MS, Erzsébet VO, Vargha P and Kempler P: Heart rate variability is severely impaired among type 2 diabetic patients with hypertension. Diabetes Metab Res Rev 30: 305-312, 2014.

13 Nenna A, Lusini M, Spadaccio C, Nappi F, Greco SM, Barbato R, Covino E and Chello M: Heart rate variability: A new tool to predict complications in adult cardiac surgery. J Geriatr Cardiol 14: 662-668, 2003. 
14 Hejjel L and Roth E: What is the adequate sampling interval of the ECG signal for heart rate variability analysis in the time domain? Physiol Meas 25: 1405-1411, 2004.

15 Toth V, Hejjel L, Fogarasi A, Gyimesi C, Orsi G, Szucs A, Kovacs N, Komoly S, Ebner A and Janszky J: Periictal heart rate variability analysis suggests long-term postictal autonomic disturbance in epilepsy. Eur J Neurol 17: 780-787, 2010.

16 Klintworth A, Ajtay Z, Paljunite A, Szabados S and Hejjel L: Heart rate asymmetry follows the inspiration/expiration ratio in healthy volunteers. Physiol Meas 33: 1717-1731, 2012.

17 Task Force of the European Society of Cardiology the North American Society of Pacing Electrophysiology: Heart Rate Variability Standards of Measurement, Physiological Interpretation, and Clinical Use. Circulation 93: 1043-1065, 1996.
18 Németh B, Kiss I, Jencsik T, Péter I, Kreska Z, Kőszegi T, Miseta A, Kustán P, Boncz I, Laczo A and Ajtay Z: Angiotensinconverting enzyme inhibition improves the effectiveness of transcutaneous carbon dioxide treatment. In Vivo 31: 425-428, 2017.

19 Bickel A, Yahalom M, Roguin N, Frankel R, Breslava J, Ivry S and Eitan A: Power spectral analysis of heart rate variability during positive pressure pneumoperitoneum: the significance of increased cardiac sympathetic expression. Surg Endosc 16: 1341-1344, 2002.

Received May 31, 2018

Revised June 19, 2018

Accepted June 20, 2018 